\title{
Palladium-Catalyzed, Copper(I)-Mediated Coupling of Boronic Acids and Benzylthiocyanate. A Cyanide-Free Cyanation of Boronic Acids
}

\author{
Zhihui Zhang and Lanny S. Liebeskind* \\ Department of Chemistry, Emory University, 1515 Dickey Drive, Atlanta, Georgia 30322
}

\section{Supporting Information}

\section{General Methods}

All reactions were performed under an atmosphere of dry argon in oven-dried glassware. THF, DMA, DMF, NMP, toluene and 1,4-dioxane were dried over $4 \AA$ molecular sieves and titrated for water level prior to use with a Fisher Coulomatic K-F titrater. $\mathrm{Et}_{3} \mathrm{~N}$ was dried over $\mathrm{KOH}$ pellets. Hexanes, ethyl acetate (EtOAc), and diethyl ether $\left(\mathrm{Et}_{2} \mathrm{O}\right)$ were obtained from EM Science and used as purchased. 'Brine' refers to a saturated aqueous solution of $\mathrm{NaCl}$. Unless otherwise specified, solutions $\mathrm{NH}_{4} \mathrm{Cl}$ and $\mathrm{Na}_{2} \mathrm{CO}_{3}$ refer to aqueous solutions. Purification by flash chromatography was performed using Whatman $60 \AA 230-400$ mesh $\mathrm{SiO}_{2}$ with compressed air as a source of positive pressure. Purification by plate chromatography was performed on EM Science Kieselgel $0.5 \mathrm{~mm}$ or $1 \mathrm{~mm} 60 \mathrm{~F}_{254}$ plates. Analytical thin-layer chromatography (TLC) was carried out using Merck Kieselgel $60 \mathrm{~F}_{254}$ plates with visualization by UV or phosphomolybdic acid.

${ }^{1} \mathrm{H}$ NMR spectra were recorded on a INOVA $400 \mathrm{MHz}$ NMR spectrometer at room temperature in $\mathrm{CDCl}_{3}$ and were internally referenced to $\mathrm{CDCl}_{3}(7.27 \mathrm{ppm}) ;{ }^{13} \mathrm{C}$ NMR spectra were recorded on a $100 \mathrm{MHz} \mathrm{NMR}$ spectrometer at room temperature in $\mathrm{CDCl}_{3}$ and were 
internally referenced to $\mathrm{CDCl}_{3}(77.23 \mathrm{ppm})$. Data are reported in the following order: chemical shifts are given ( $\delta$ ); multiplicities are indicated (br (broadened), s (singlet), d (doublet), $\mathrm{t}$ (triplet), q (quartet), hex (hextet), hept (heptet), m (multiplet), exch (exchangeable), app (apparent)); coupling constants, $J$, are reported $(\mathrm{Hz})$; integration is provided. Infrared spectra were recorded ASI ReactIR 1000FT-IR spectrometer with a silicone probe. Peaks are reported $\left(\mathrm{cm}^{-1}\right)$ with the following relative intensities: $\mathrm{s}$ (strong, 67-100\%), m (medium, 40-67\%), w (weak, 20-40\%) and br (broad). GC-MS spectra were recorded on a Shimadzu Gas Chromatograph GC-17A, Mass Spectrometer QP-5000. GC/MS analysis was carried out on a bonded 5\% diphenylsiloxane capillary column $(30 \mathrm{~m}, 0.25 \mathrm{~mm}$ id, $0.25 \mu \mathrm{m} \mathrm{df}$ ). Melting points were taken on a Thomas-Hoover melting point apparatus in open capillary tubes, and were not corrected.

\section{Starting Materials}

Benzenethiol, p-nitrophenylthiol, triethylamine, sulfuryl chloride, trimethylsilyl cyanide, benzyl thiocyanate, ethylthiocyanate, $\mathrm{Pd}\left(\mathrm{PPh}_{3}\right)_{4}$ were all purchased from Aldrich and used without further purification. All boronic acids were purchased from Frontier Scientific, Inc. Copper(I) thiophene-2-carboxylate(CuTC), ${ }^{1}$ phenylthiocyanate and p-nitrophenylthiocyanate ${ }^{2}$ were prepared following literature procedures.

\section{General Procedure for Thiocyanate - Boronic Acid Cross-Coupling Reaction.}

Benzylthiocyanate, the boronic acid (1.5 equiv), CuTC (1.5-3.0 equiv), and $\mathrm{Pd}\left(\mathrm{PPh}_{3}\right)_{4}(3 \mathrm{~mol}$ $\%$ ) were added into a flask that was then flushed with argon. Dry and degassed 1,4-dioxane

\footnotetext{
${ }^{1}$ Allred, G.; Liebeskind, L. S. J. Am. Chem. Soc. 1996, 118, 2748.

${ }^{2}$ Still, Ian W. J.; Watson, Iain D. G. Synth. Commun. 2001, 31, 1355.
} 
was added ( $2 \mathrm{~mL}$ for each $0.1 \mathrm{mmol}$ thiocyanate). The brown suspension was stirred under

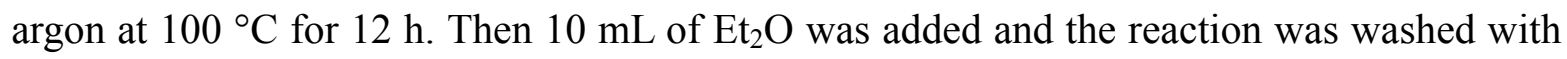
satd $\mathrm{NH}_{4} \mathrm{Cl}$ followed by satd $\mathrm{Na}_{2} \mathrm{CO}_{3}$ and then brine. After evaporation of the solvent, the residue was purified by preparative plate silica chromatography $\left(\mathrm{SiO}_{2}, 4: 1\right.$ hexanes/EtOAc) to give the desired product.

Benzonitrile (Table 1, entry 1$)^{3}$

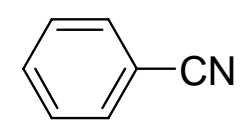

Colorless oil; ${ }^{1} \mathrm{H}$ NMR $\left(400 \mathrm{MHz}, \mathrm{CDCl}_{3}\right) \delta 7.67 \sim 7.59(\mathrm{~m}, 3 \mathrm{H}), 7.50 \sim 7.46(\mathrm{~m}, 2 \mathrm{H}) ;{ }^{13} \mathrm{C}$ NMR (100 MHz, $\left.\mathrm{CDCl}_{3}\right) \delta 133.0,132.3,129.3,119.0,112.6$; IR (neat, $\left.\mathrm{cm}^{-1}\right): 2231(\mathrm{~s})$.

Naphthalene-2-carbonitrile (Table 1, entry 2) ${ }^{4}$<smiles>N#Cc1ccc2ccccc2c1</smiles>

White solid; $\mathrm{Mp} 65-66^{\circ} \mathrm{C}\left(\right.$ lit. $\left.\left\{65-66^{\circ} \mathrm{C}\right\}^{4}\right) .{ }^{1} \mathrm{H}$ NMR $\left(400 \mathrm{MHz}, \mathrm{CDCl}_{3}\right) \delta 8.23(\mathrm{~d}, J=0.8$ $\mathrm{Hz}, 1 \mathrm{H}), 7.92 \sim 7.88(\mathrm{~m}, 3 \mathrm{H}) ; 7.67 \sim 7.59(\mathrm{~m}, 3 \mathrm{H}) ;{ }^{13} \mathrm{C} \mathrm{NMR}\left(100 \mathrm{MHz}, \mathrm{CDCl}_{3}\right) \delta 134.8,134.3$, 132.4, 129.4, 129.2, 128.6, 128.2, 127.8, 126.5, 119.4, 109.5; IR (neat, $\left.\mathrm{cm}^{-1}\right): 2227(\mathrm{~s})$.

Naphthalene-1-carbonitrile (Table 1 , entry 3$)^{5}$<smiles>N#Cc1cccc2ccccc12</smiles>

White solid; Mp 34-35 ${ }^{\circ} \mathrm{C}$ (lit. $\left.\left\{33-34{ }^{\circ} \mathrm{C}\right\}{ }^{5}\right) .{ }^{1} \mathrm{H}$ NMR $\left(400 \mathrm{MHz}, \mathrm{CDCl}_{3}\right) \delta 8.26(\mathrm{~d}, J=8.4$ $\mathrm{Hz}, 1 \mathrm{H}), \delta 8.10(\mathrm{~d}, J=8.4 \mathrm{~Hz}, 1 \mathrm{H}), 7.95 \sim 7.92(\mathrm{~m}, 2 \mathrm{H}), 7.73 \sim 7.69(\mathrm{~m}, 1 \mathrm{H}), 7.65 \sim 7.61(\mathrm{~m}, 1 \mathrm{H})$,

${ }^{3}$ Fung, B. M. J. Am. Chem. Soc. 1983, 105, 5713.

${ }^{4}$ House, H. O; Fisher, W. F. J. Org. Chem. 1969, 34, 3626.

${ }^{5}$ Friedman, L.; Shechter, H. J. Org. Chem. 1961, 25, 2522. 
$7.55 \sim 7.52(\mathrm{~m}, 1 \mathrm{H}) ;{ }^{13} \mathrm{C} \mathrm{NMR}\left(100 \mathrm{MHz}, \mathrm{CDCl}_{3}\right) \delta 133.5,133.1,132.8,132.5,128.9,128.8$, 127.7, 125.3, 125.1, 118.0, 110.4; IR (neat, $\mathrm{cm}^{-1}$ ): 2223 (s).

4-(Dimethylamino)benzonitrile (Table 1, entry 4) ${ }^{6}$

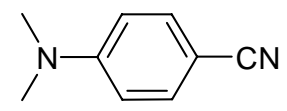

Blue solid; Mp 75-76 $\left.{ }^{\circ} \mathrm{C}\left(\text { lit. }\left\{75-77^{\circ} \mathrm{C}\right\}\right\}^{6}\right) .{ }^{1} \mathrm{H}$ NMR $\left(400 \mathrm{MHz}, \mathrm{CDCl}_{3}\right) \delta 7.49(\mathrm{~d}, J=8.8 \mathrm{~Hz}$, 2H), $6.66(\mathrm{~d}, J=8.8 \mathrm{~Hz}, 2 \mathrm{H}) ; 3.04(\mathrm{~s}, 6 \mathrm{H}) ;{ }^{13} \mathrm{C} \mathrm{NMR}\left(100 \mathrm{MHz}, \mathrm{CDCl}_{3}\right) \delta 152.6,133.5$, 121.0, 111.6, 97.5, 40.2; IR (neat, $\left.\mathrm{cm}^{-1}\right): 2212$ (s).

2, 5-Dimethoxybenzonitrile (Table 1, entry 5) ${ }^{7}$

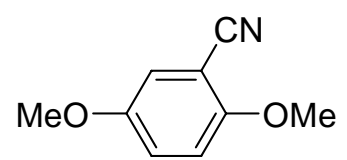

White solid; Mp 79-80 ${ }^{\circ} \mathrm{C}\left(\right.$ lit. $\left.\left\{80-81{ }^{\circ} \mathrm{C}\right\}{ }^{7}\right) .{ }^{1} \mathrm{H}$ NMR $\left(400 \mathrm{MHz}, \mathrm{CDCl}_{3}\right) \delta 7.11(\mathrm{~m}, 2 \mathrm{H}), 6.92$ $(\mathrm{d}, J=9.2 \mathrm{~Hz}, 1 \mathrm{H}), 3.89(\mathrm{~s}, 3 \mathrm{H}), 3.78(\mathrm{~s}, 3 \mathrm{H}) .{ }^{13} \mathrm{C} \mathrm{NMR}\left(100 \mathrm{MHz}, \mathrm{CDCl}_{3}\right) \delta 155.9,153.3$, 121.0, 117.7, 116.6, 112.8, 102.0, 56.6, 56.1; IR (neat, $\left.\mathrm{cm}^{-1}\right): 2227$ (s).

\section{2,3,4-Trimethoxybenzonitrile (Table 1, entry 6) ${ }^{8}$}

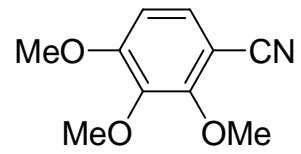

White solid; Mp 55-56 ${ }^{\circ} \mathrm{C}$ (lit. $\left.\left.\left\{52-55{ }^{\circ} \mathrm{C}\right\}\right\}^{8}\right) .{ }^{1} \mathrm{H}$ NMR $\left(400 \mathrm{MHz}, \mathrm{CDCl}_{3}\right) \delta 7.29(\mathrm{~d}, J=8.8$ $\mathrm{Hz}, 1 \mathrm{H}), 6.69(\mathrm{~d}, J=8.8 \mathrm{~Hz}, 1 \mathrm{H}), 4.05$ (s, 3H), 3.91 (s, 3H), $3.86(\mathrm{~s}, 3 \mathrm{H}) .{ }^{13} \mathrm{C} \mathrm{NMR}(100 \mathrm{MHz}$, $\left.\mathrm{CDCl}_{3}\right) \delta 158.2,156.1,142.1,129.0,107.7,107.0,99.3,62.0,61.3,56.5$; IR (neat, $\mathrm{cm}^{-1}$ ): $2227(\mathrm{~s})$

${ }^{6}$ Chen, F.; Kuang. Y.; Dai. H.; Lu. L.; Huo. M. Synthesis 2003, 2629.

${ }^{7}$ Wallenfels, K.; Hofmann, D.; Kern, R. Tetrahedron 1965, 21, 2231.

${ }^{8}$ Kovach, E. G.; Barnes, D. E. J. Am. Chem. Soc. 1954, 76, 1176. 
2,4,6-Trimethyl-benzonitrile (Table 1, entry 7) ${ }^{9}$

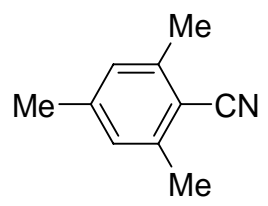

White solid; Mp 50-51 ${ }^{\circ} \mathrm{C}$ (lit. $\left.\left\{50-52{ }^{\circ} \mathrm{C}\right\}{ }^{9}\right) .{ }^{1} \mathrm{H}$ NMR $\left(400 \mathrm{MHz}, \mathrm{CDCl}_{3}\right) \delta 6.93(\mathrm{~s}, 2 \mathrm{H}), 2.48$ (s, 6H), $2.32(\mathrm{~s}, 3 \mathrm{H}) ;{ }^{13} \mathrm{C} \mathrm{NMR}\left(100 \mathrm{MHz}, \mathrm{CDCl}_{3}\right) \delta 143.0,142.2,128.4,117.8,110.5,21.8$, 20.8; IR (neat, $\mathrm{cm}^{-1}$ ): $2216(\mathrm{~s})$.

3-Acetylbenzonitrile (Table 1 , entry 8) ${ }^{10}$<smiles>CC(=O)c1cccc(C#N)c1</smiles>

White solid; Mp 97-98 ${ }^{\circ} \mathrm{C}\left(\right.$ lit. $\left.\left\{98^{\circ} \mathrm{C}\right\}{ }^{10}\right) .{ }^{1} \mathrm{H}$ NMR (400 MHz, $\left.\mathrm{CDCl}_{3}\right) \delta 8.24 \sim 8.18(\mathrm{~m}, 2 \mathrm{H})$, 7.86 7.84 (m, 1H), 7.64 7.60 (m, 1H), $2.65(\mathrm{~s}, 3 \mathrm{H}) ;{ }^{13} \mathrm{C} \mathrm{NMR}\left(100 \mathrm{MHz}, \mathrm{CDCl}_{3}\right) \delta 195.8$, 137.6, 135.9, 132.1, 131.8, 129.7, 117.7, 113.0, 26.7; IR (neat, $\mathrm{cm}^{-1}$ ): 2235 (m), 1683 (s).

4-Cyanobenzoic acid methyl ester (Table 1, entry 9) ${ }^{11}$

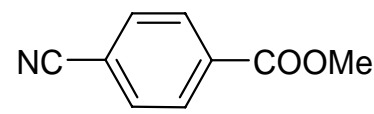

White solid; Mp 65-66 ${ }^{\circ} \mathrm{C}$ (lit. $\left.\left\{67-68^{\circ} \mathrm{C}\right\}^{11}\right) .{ }^{1} \mathrm{H}$ NMR $\left(400 \mathrm{MHz}, \mathrm{CDCl}_{3}\right) \delta 8.15(\mathrm{~m}, 2 \mathrm{H})$, $7.76(\mathrm{~m}, 2 \mathrm{H}), 3.96(\mathrm{~s}, 3 \mathrm{H}) ;{ }^{13} \mathrm{C} \mathrm{NMR}\left(100 \mathrm{MHz}, \mathrm{CDCl}_{3}\right) \delta 165.6,134.1,132.4,130.3,118.1$, 116.6, 52.9; IR (neat, $\left.\mathrm{cm}^{-1}\right): 2231(\mathrm{~m}), 1725(\mathrm{~s})$.

4-Chlorobenzonitrile (Table 1, entry 10) ${ }^{12}$

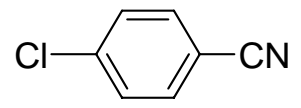

\footnotetext{
${ }^{9}$ Rutan, K. J.; Heldrich, F. J.; Borges, L. F. J .Org. Chem. 1995, 60, 2948.

${ }^{10}$ Yamada, H.; Tsuno, Y.; Yukawa, Y. Bull. Chem. Soc. Jpn. 1970, 43, 1459.

11 Takagi, K.; Sasaki, K.; Sakakibara, Y. Bull. Chem. Soc. Jpn. 1991, 64, 1118.
} 
White solid; Mp 91-93 ${ }^{\circ} \mathrm{C}\left(\right.$ lit. $\left.\left\{90.5-91.5{ }^{\circ} \mathrm{C}\right\}{ }^{12}\right) .{ }^{1} \mathrm{H}$ NMR $\left(400 \mathrm{MHz}, \mathrm{CDCl}_{3}\right) \delta$ 7.62 7.60 (m, 1H), 7.48 7.46 (m, 1H); ${ }^{13} \mathrm{C}$ NMR $\left(100 \mathrm{MHz}, \mathrm{CDCl}_{3}\right) \delta$ 139.7, 133.6, 129.9, 118.2, 110.9; IR (neat, $\left.\mathrm{cm}^{-1}\right): 2231(\mathrm{~s})$.

\section{1,4-Dicyanobenzene (Table 1, entry 11) ${ }^{13}$}

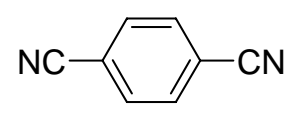

White solid; Mp 223-224 ${ }^{\circ} \mathrm{C}\left(\right.$ lit. $\left.\left\{226-227^{\circ} \mathrm{C}\right\}^{13}\right) .{ }^{1} \mathrm{H}$ NMR $\left(400 \mathrm{MHz}, \mathrm{CDCl}_{3}\right) \delta 7.80(\mathrm{~s}, 4 \mathrm{H})$;

${ }^{13} \mathrm{C}$ NMR $\left(100 \mathrm{MHz}, \mathrm{CDCl}_{3}\right) \delta$ 133.0, 117.2, 116.9; IR (neat, $\left.\mathrm{cm}^{-1}\right): 2227$ (s).

3-Cyanothiophene (Table 1 , entry 13) ${ }^{14}$

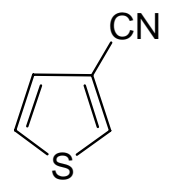

Light yellow oil; ${ }^{1} \mathrm{H}$ NMR (400 MHz, $\left.\mathrm{CDCl}_{3}\right) \delta 7.96(\mathrm{dd}, J=3.2,1.2 \mathrm{~Hz}, 1 \mathrm{H}), 7.45(\mathrm{dd}, J=$

5.2, 3.2 Hz, 1H), $7.32(\mathrm{dd}, J=5.2,1.2 \mathrm{~Hz}, 1 \mathrm{H}) ;{ }^{13} \mathrm{C} \mathrm{NMR}\left(100 \mathrm{MHz}, \mathrm{CDCl}_{3}\right) \delta$ 135.6, 128.9, 127.5, 115.3, 110.9; IR (neat, $\left.\mathrm{cm}^{-1}\right): 2231(\mathrm{~s})$.

2-Methoxy-4-cyanopyridine (Table 1 , entry 14)

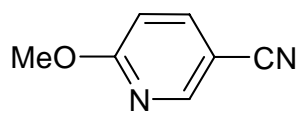

White solid; Mp 95-96 ${ }^{\circ} \mathrm{C}\left(\right.$ lit. $\left.\left\{97^{\circ} \mathrm{C}\right\}{ }^{15}\right) .{ }^{1} \mathrm{H}$ NMR $\left(400 \mathrm{MHz}, \mathrm{CDCl}_{3}\right) \delta 8.49(\mathrm{~d}, J=2.4 \mathrm{~Hz}$, 1H), $7.79(\mathrm{dd}, J=8.8,2.4 \mathrm{~Hz}, 2 \mathrm{H}), 6.83(\mathrm{~d}, J=8.8 \mathrm{~Hz}, 1 \mathrm{H}), 3.99(\mathrm{~s}, 3 \mathrm{H}) ;{ }^{13} \mathrm{C}$ NMR $(100$ $\left.\mathrm{MHz}, \mathrm{CDCl}_{3}\right) \delta 166.25,152.2,141.0,117.5,112.0,102.6,54.5$; IR (neat, $\mathrm{cm}^{-1}$ ): 2231(s),

\footnotetext{
12 Murahashi, S.; Naota, T.; Nakajima, N. J. Org. Chem. 1986, 51, 898.

${ }_{13}$ Mulvaney, J. E.; Marvel, C. S. J. Org. Chem. 1961, 26, 95.

14 Terui, Y.; Yamakawa, M.; Honma, T.; Yada, Y.; Tori, K. Heterocycles 1982, 19, 221.

15 Khanna, I. K.; Yu, Y.; Huff, R. M.; Weier, R. M.; Xu, X.; Koszyk, F. J.; Collins, P. W.; Cogburn, J. N.; Isakson, P. C.; Koboldt, C. M.; Masferrer, J. L.; Perkins, W. E.; Seibert, K.; Veenhuizen, A. W.; Yuan, J.; Yang, D-C.; Zhang, Y. Y. J. Med. Chem. 2000, 43, 3168.
} 
$1602(s)$.

(E)-3-Phenyl-2-propenonitrile (Table 1, entry 15) ${ }^{16}$<smiles>N#C/C=C/c1ccccc1</smiles>

Colorless oil. ${ }^{1} \mathrm{H}$ NMR $\left(400 \mathrm{MHz}, \mathrm{CDCl}_{3}\right) \delta$ 7.46 7.39 (m, 6H), $5.85(\mathrm{~d}, J=16.4 \mathrm{~Hz}, 1 \mathrm{H})$;

${ }^{13} \mathrm{C}$ NMR $\left(100 \mathrm{MHz}, \mathrm{CDCl}_{3}\right) \delta 150.8,133.7,131.4,129.3,127.5,118.3,96.5$. IR (neat, $\left.\mathrm{cm}^{-1}\right)$ : 2219(s).

(E)-4-Phenyl-2-butenonitrile (Table 1, entry 16) ${ }^{17}$<smiles>N#C/C=C/Cc1ccccc1</smiles>

Colorless oil. ${ }^{1} \mathrm{H}$ NMR $\left(400 \mathrm{MHz}, \mathrm{CDCl}_{3}\right) \delta$ 7.36 7.14 (m, 5H), $6.92(\mathrm{dt}, J=16.4,6.4 \mathrm{~Hz}$, 1H), $5.31(\mathrm{dt}, J=16.4,1.6 \mathrm{~Hz}, 1 \mathrm{H}), 3.55(\mathrm{dd}, J=6.4,1.6 \mathrm{~Hz}, 2 \mathrm{H}) ;{ }^{13} \mathrm{C}$ NMR $(100 \mathrm{MHz}$, $\left.\mathrm{CDCl}_{3}\right) \delta 154.3,136.2,129.1,129.0,127.4,117.5,101.0,39.5$; IR (neat, $\left.\mathrm{cm}^{-1}\right): 2223(\mathrm{~s})$.

${ }^{16}$ Hamza, K.; Abu-Reziq, R.; Avnir, D.; Blum, J. Org. Lett. 2004, 6, 925.

${ }^{17}$ Inaba, S.; Matsumoto, H.; Rieke, R. J. Org. Chem. 1984, 49, 2093. 